and injury. J. Clin. Invest. 114:495-503. doi:10.1172/ JCI200419297.

9. Mu, J., Brozinick, J.T., Valladares, O., Bucan, M., and Birnbaum, M.J. 2001. A role for AMP-activated protein kinase in contraction- and hypoxia-regulated glucose transport in skeletal muscle. Mol. Cell. 7:1085-1094.

10. Hudson, E.R., et al. 2003. A novel domain in AMPactivated protein kinase causes glycogen storage bodies similar to those seen in hereditary cardiac arrhythmias. Curr. Biol. 13:861-866.

11. Polekhina, G., et al. 2003. AMPK b-Subunit targets metabolic stress-sensing to glycogen. Curr. Biol. 13:867-871.

12. Scott, J.W., et al. 2004. CBS domains form energysensing modules whose binding of adenosine ligands is disrupted by disease mutations. J. Clin.
Invest. 113:274-284. doi:10.1172/JCI200419874.

13. Xing, Y., et al. 2003. Glucose metabolism and energy homeostasis in mouse hearts overexpressing dominant negative alpha2 subunit of AMP-activated protein kinase. J. Biol. Chem. 278:28372-28377.

14. Mangano, D.T. 1997. Effects of acadesine on myocardial infarction, stroke, and death following surgery. A meta-analysis of the 5 international randomized trials. The Multicenter Study of Perioperative Ischemia (McSPI) Research Group. JAMA. 277:325-332.

15. Gadalla, A.E., et al. 2004. Distinct mechanisms underlie the activation of rat brain AMP-activated protein kinase and the inhibition of excitatory synaptic transmission by AICA riboside (Acadesine) in area CA1 of rat hippocampus. J. Neurochem. 88:1272-1282.

16. Corton, J.M., Gillespie, J.G., Hawley, S.A., and Har- die, D.G. 1995. 5-Aminoimidazole-4-carboxamide ribonucleoside: a specific method for activating AMP-activated protein kinase in intact cells? Eur. J. Biochem. 229:558-565.

17. Javaux, F., Vincent, M.F., Wagner, D.R., and van den Berghe, G. 1995. Cell-type specificity of inhibition of glycolysis by 5-amino-4-imidazolecarboxamide riboside. Lack of effect in rabbit cardiomyocytes and human erythrocytes, and inhibition in FTO2B rat hepatoma cells. Biochem. J. 305:913-919.

18. Andersson, U., et al. 2004. AMP-activated protein kinase plays a role in the control of food intake. J. Biol. Chem. 279:12005-12008.

19. Minokoshi, Y., et al. 2004. AMP-kinase regulates food intake by responding to hormonal and nutrient signals in the hypothalamus. Nature. 428:569-574

\title{
Immune recognition of self in immunity against cancer
}

\author{
Alan N. Houghton and José A. Guevara-Patiño
}

The Swim Across America Laboratory of Tumor Immunology, Memorial Sloan-Kettering Cancer Center, New York, New York, USA.

\begin{abstract}
Most antigens expressed by human cancer cells and recognized by host $T$ cells and antibodies are nonmutated self antigens - molecules also expressed on the surface of normal cells. These self antigens are ineffective at triggering immune responses against cancer cells, which provides one explanation for the difficulties in trying to immunize against human cancer. A new study describes how tumors can avoid recognition by the immune system and how enhancing the affinity of the interaction between a self antigen and the MHC-I molecule may lead to cancer immunity (see the article beginning on page 551).
\end{abstract}

\section{The self/nonself paradigm}

Immunologists generally agree that the immune system was shaped through evolution by the necessity of discriminating nonself pathogens from self tissues (1). This distinction is extremely important for the survival of multicellular organisms. Some strategies for the recognition of nonself pathogens are clear-cut. For example, receptors such as toll-like receptors on host leukocytes bind to certain bacterial or fungal molecules, leading to mobilization of host defenses by signaling the synthesis of molecules that initiate innate and adaptive immune responses. The adaptive immune system, with its diversity of $\mathrm{T}$ cell receptors (TCRs) and antibodies, uses even more precise mechanisms for the recognition of nonself pathogens. This view of self/nonself

Nonstandard abbreviations used: $T$ cell receptor (TCR).

Conflict of interest: The authors have declared that no conflict of interest exists.

Citation for this article:

J. Clin. Invest. 114:468-471 (2004).

doi:10.1172/JCI200422685 provides a logical and useful description of how the immune system responds to exogenous microbes that cause disease.

\section{Recognition of cancer by the host's immune system}

Understanding and achieving immunity to cancer does not fit neatly into the self/ nonself paradigm because cancer is not an exogenous pathogen, but rather arises from normal host cells. In this regard, cancer antigens recognized by the human immune system are self or mutated self molecules (2). Explaining the genetic basis for the pathogenesis of cancer is necessary to understanding the difficulties that the immune system has in recognizing cancer cells.

Protooncogenes and tumor suppressor genes are normal cellular genes that control crucial cell functions, particularly growth and survival (3). Mutations in these genes lead to the emergence of cancer. In addition, loss of expression of tumor suppressor genes contributes to malignant transformation through major deletions in their genetic sequences or silencing by methylation of nucleotides in promoter regions that control gene expression. These deleted or silent genes are incapable of producing antigenic targets for the immune system, but mutated protooncogenes, tumor suppressor genes, or other self genes might generate mutant protein products that could serve as suitable antigens.

An accumulation of genetic alterations in protooncogenes and tumor suppressor genes leads to profound changes in normal cells, including immortality, a block in terminal differentiation, an ability to invade normal tissues and recruit new blood vessels, and the potential to metastasize to distant organs (3). However, because the immune system is trained not to respond to self molecules (in order to avoid autoimmunity), antigenic changes in malignant cells that are created by individual mutations can be rather subtle from the standpoint of the immune system - cancer cells still utilize essentially the same cellular molecules as healthy cells to regulate growth and survival (3). Perhaps mutations in self proteins can be viewed as generating nonself proteins, comparable to foreign proteins from pathogens or other species. This argument has been used as the basis for conducting experiments in mice for the study of tumor immunity using tumors expressing viral or chicken proteins.

$\mathrm{T}$ cells, which are immune cells crucial for rejecting tumors, use their TCRs to recognize short antigenic peptides bound 


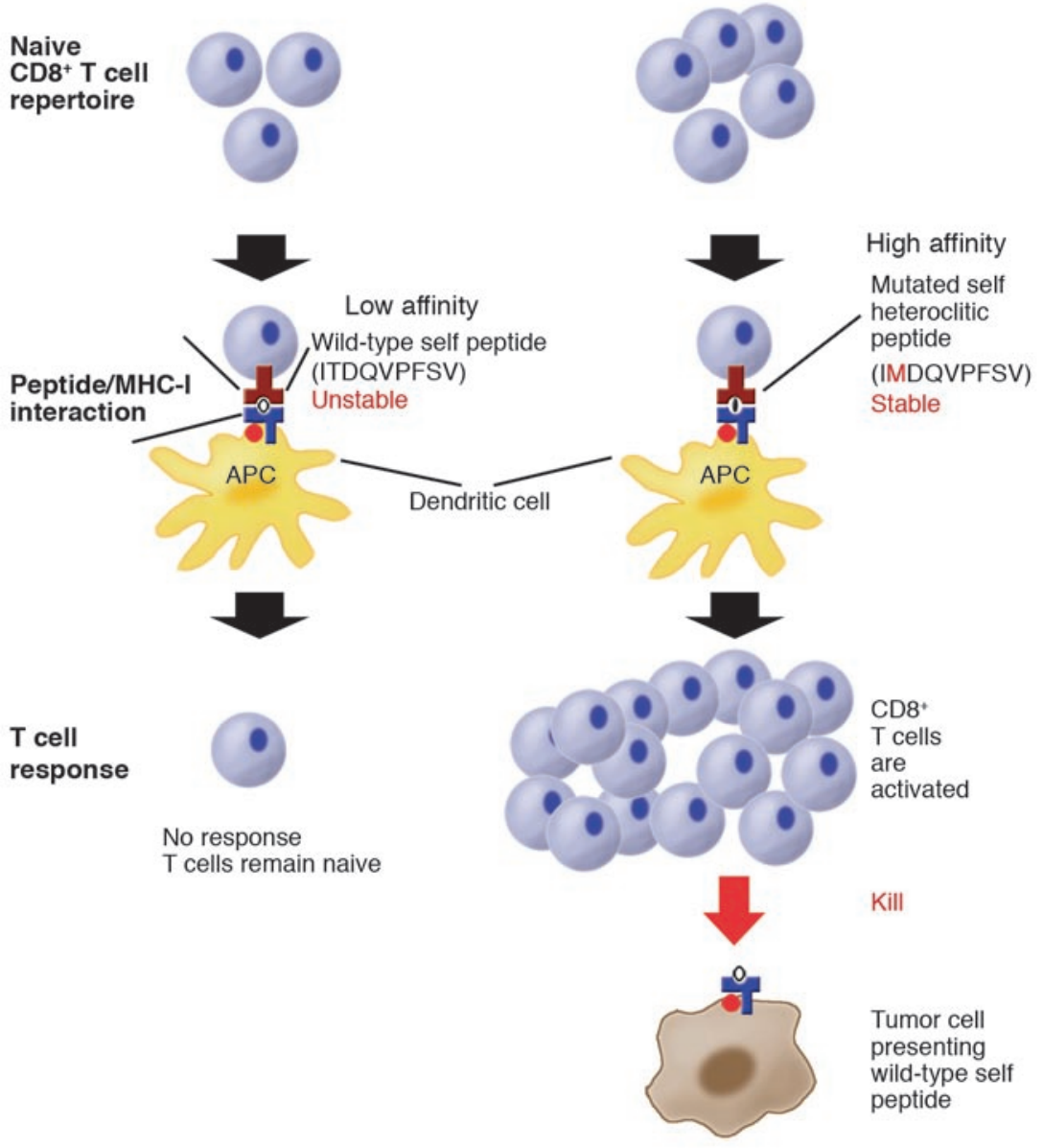

Figure 1

Yu et al. (16) investigate how host CD8+ T cells respond to a self peptide presented by MHC-I molecules on tumor cells. The self peptide binds relatively weakly to host MHC molecules. The self peptide ITDQVPFSV (single letter amino acid code), expressed by melanoma cells and normal pigment cells, binds with low affinity to MHC-I HLA-A*0201 molecules. A mutated form of the self peptide, IMDQVPFSV with a threonine to methionine substitution at the second amino acid position, has a high affinity for MHC-I molecules because methionine residues at the second position are more favorable to anchor the peptide to HLA-A ${ }^{*} 0201$ molecules. Naive CD8 ${ }^{+} T$ cells against the self peptide are present in the immune repertoire, but they do not respond to either tumor cells presenting the weak self peptide or to immunization with the self peptide because of the instability of self peptide/MHC complex. However, immunization with the mutant self peptide activates the host $\mathrm{T}$ cells. The mutant peptide is presented by MHC-I molecules on dendritic cells, which are potent APCs. Once the $\mathrm{CD} 8^{+} \mathrm{T}$ cells are activated, they are competent to recognize and kill host tumor cells presenting the nonmutated self peptide. These results show that $T$ cells recognizing a self antigen are capable of killing tumor cells presenting the self antigen following activation with the mutated form of the antigen.

to MHC-I and -II molecules on the surface of host APCs. Naive T cells that have never been activated by antigen are initially triggered by TCR recognition of specific peptide/MHC complexes presented by dendritic cells, which are specialized, professional APCs that provide additional potent costimulatory signals for $\mathrm{T}$ cell activation. Dendritic cells capture exogenous antigens from pathogens as well as from host cells. The ability of dendritic cells to take up and present antigens is the basis for vaccination's ability to produce $\mathrm{T}$ cell immunity. Activated T cells can go on to destroy antigen-positive host cells, e.g., cells infected with pathogens or cancer cells. Host cells, including cancer cells, can serve as targets for previously activated $\mathrm{T}$ cells through the processing and presentation of antigenic peptides. However, nonprofessional APCs, including most cancer cells, which lack costimulatory molecules, are ill-equipped to initiate immune responses from naive $T$ cells.

\section{T cell-dependent immunity against mutated self antigens on tumors}

Can cancer cells with mutated self peptides stimulate host T cells? As few as two or three distinct mutations in different cellular protooncogenes can induce malignant transformation and tumorigenicity of normal rodent cells $(3,4)$. In this reductionist view, a total of only two mutations in two different cellular proteins produce tumors. The problem for host $\mathrm{T}$ cells becomes searching for these two mutations against the backdrop of the thousands of nonmutated proteins in the cell - which is like searching for a needle in a haystack. The reader may be aware that $T$ cells can use their TCRs to discriminate even single amino acid changes in peptides. Thus, activated $T$ cells might be capable of surveying for cancer cells expressing rare mutated self peptides bound to MHC-I or -II molecules, leading to destruction of those cells. In support of this argument, classical experiments in mice have shown that $\mathrm{T}$ cells are capable of rejecting mutagen-induced tumors (5). For tumors in mice induced by exposure to strong mutagens, T cell-dependent immunity is directed against antigens that are mutated self peptides $(6,7)$.

Do $T$ cell responses to mutated self antigens reveal how immunity against cancer is generated? Not exactly. Tumors that are elicited by short, intense exposure of rodents to potent carcinogens carry large numbers of mutations, and these tumors arise over weeks or several months. These experimental models do not adequately reflect the pathogenesis of most human cancers, which result from multiple discrete mutations accumulated sequentially over decades (3).

\section{Immunity against nonmutated self antigens on cancer cells}

Although the repertoire of $\mathrm{T}$ cells in humans can recognize both self and mutated self peptides on human cancer cells, $T$ cells and antibodies from cancer patients have been shown to recognize largely nonmutated self antigens (2). The widespread $T$ cell recognition of self antigens on human cancer cells is surprising. Insights into the relevance of $\mathrm{T}$ cell recognition of nonmutated self antigens have stemmed from studies of spontaneous tumors in mice. These tumors are weakly immunogenic, yet arguably are more representative of most human cancers than the strongly immunogenic mutagen-induced tumors $(5,8)$. 
$\mathrm{T}$ cells can reject weakly immunogenic spontaneous tumors by recognizing nonmutated self antigens as long as special strategies are used for immunization (913). How could $T$ cells be triggered to reject tumors expressing weak self antigens? The answer starts with understanding how the repertoire of host $\mathrm{T}$ cells is selected during development. Any immature T cell with high-affinity TCRs for a self antigen is deleted during its early development in the thymus; this process leads to destruction of more than $90 \%$ of immature T cells. Elimination of these high-affinity $\mathrm{T}$ cells is necessary to avoid the development of autoimmunity. However, despite this stringent selection, the immune repertoire is replete with mature, naive, self-reactive $T$ cells that have TCRs with relatively low affinity for self antigens. These weak self antigens are incapable of inducing immune responses. However, if T cells against these apparently ineffective self antigens can somehow be activated through vaccination or other means, then these $T$ cells can reject tumors presenting these antigens (9-13).

\section{A role for mutated peptides in eliciting immunity against nonmutated self antigens}

Experimental evidence in mice has previously shown that poorly immunogenic spontaneous tumors can be converted to strongly immunogenic tumors by intense exposure to mutagens $(14,15)$. Remarkably, immunity against mutagenized tumors also yields $T$ cell-dependent immunity against the parental nonmutagenized tumor. How might mutations in self peptides induce $\mathrm{T}$ cell responses against weak self antigens? One potential mechanism relies on point mutations that enhance peptide binding to MHC-I molecules, leading to immunity against the nonmutated self peptides presented by tumor cells (Figure 1). The paper by Yu et al. in this issue of the JCI demonstrates the relevance of this mechanism to cancer immunity (16).

Selected missense mutations in self peptides increase peptide binding to MHC-I or-II molecules by altering single amino acid residues that anchor the peptide to the $\mathrm{MHC}$ molecule. Enhanced binding of the mutant peptides to $\mathrm{MHC}$ molecules provides more favorable energy conditions at the APC/TCR synapse for $\mathrm{T}$ cell activation. These mutations increase the cell surface density of self peptides at this synapse on the surface of APCs through increased affinity for MHC molecules (Figure 1). These missense muta- tions are not really nonself, but rather have been termed altered self (17).

Some missense mutations in self peptides increase TCR signaling sufficiently to trigger activation of naive $T$ cells (Figure 1). Once these $T$ cells are primed, they may respond to the parental nonmutant peptide presented by cancer cells and normal cells. Such antigens have been called heteroclitic. Previous experiments have shown that vaccination with a mutant form of a nonimmunogenic peptide, rationally designed to enhance binding to MHC-I molecules, leads to T cell-dependent rejection of tumors expressing the parental nonmutant peptide (18). Furthermore, other reports have shown that vaccination using molecules from other species, specifically immunizing with polypeptides to host MHC-I molecules, generates $\mathrm{T}$ cell responses against poorly immunogenic spontaneous tumors expressing only nonmutated self antigens (9-13).

\section{Host T cells ignore cancer cells expressing weak self antigens}

In this issue of the JCI, Yu and colleagues provide important insights into how tumor cells expressing a weak self antigen avoid recognition by host $\mathrm{T}$ cells (16). Three possible mechanisms for tumor escape from $T$ cell immunity are explored: (a) insufficient numbers of host $\mathrm{T}$ cells against the self antigen are present in the $\mathrm{T}$ cell repertoire; (b) immune tolerance of $\mathrm{T}$ cells occurs through anergy (a permanent state of T cell inactivation), $\mathrm{T}$ cell deletion, or suppression by host regulatory cells; or (c) naive $\mathrm{T}$ cells against the self peptide ignore antigenpositive cells because of inadequate affinity of self peptide for host MHC molecules.

To address these possibilities, $\mathrm{Yu}$ and colleagues have created a remarkable strain of transgenic mice engineered to express both human TCR chains against an antigenic peptide in T cells and human MHC-I domains broadly in somatic cells (16). The antigenic peptide recognized by the human TCRs is expressed by both human and mouse melanoma cells and melanocytes (normal pigment cells in the epidermis and other tissues). Because the MHC-I domains, TCR chains, and antigenic peptide are of human origin, this humanized mouse system has direct relevance to the generation of $T$ cell immunity against human melanoma. The 9 amino acid, wild-type self peptide has low affinity for MHC-I molecules and elicits minimal or no responses in naive $\mathrm{T}$ cells. In contrast, a heteroclitic mutated peptide, which contains a single amino acid substitution that generates a 6 -fold improved affinity of the peptide for MHC-I molecules, activates naive host $\mathrm{T}$ cells (Figure 1). Tumor rejection only occurs following immunization with heteroclitic peptide; wild-type peptide is ineffective.

This report demonstrates that improvements in the affinity of the peptide/MHC-I interaction can overcome the barrier to priming $\mathrm{T}$ cell-mediated immunity against weak self antigens. In this system, naive $T$ cells expressing TCRs against wild-type self peptide ignore melanoma cells presenting this peptide because of the low affinity of the peptide for MHC-I molecules. The authors clearly show that this lack of $\mathrm{T}$ cell responsiveness is not due to insufficient numbers of $\mathrm{T}$ cells, $\mathrm{T}$ cell deletion, anergy, or other tolerance mechanisms (16). These results have relevance for development of experimental vaccines for the treatment of human cancer, demonstrating that careful attention needs to be paid to the affinity of potential antigenic peptides for MHC molecules.

\section{Concluding remarks}

Overcoming the barriers to the generation of immunity against self and mutated self antigens is a central concern for the ongoing efforts to treat cancer using active immunization. Inducing immunity against self antigens is dangerous territory because of the potential for autoimmune damage to crucial normal tissues. However, treatments using passive immunization with monoclonal antibodies against self antigens have already been successful in objectively shrinking established tumors in patients with cancer, without serious toxicities (e.g., the administration of rituximab, which is active against CD20 on the surface of B cells for the treatment of patients with lymphoma) $(19,20)$.

The self/nonself paradigm describes immunity against microbial pathogens but does not adequately portray adaptive immunity to cancer. We have proposed a self/altered-self paradigm as a more appropriate description of immune responses to self and mutated self antigens on cancer cells (17). The results of Yu et al. and other reports raise a series of questions $(6,7,9-16)$. How frequently are mutations recognized? What types of mutations enhance $T$ cell responses against nonmutated self peptides? How might the immune system have evolved to recognize not only pathogens but also cancer cells (in the context of self/altered self)? We believe that the answers to these questions are important to understanding cancer immunity and perhaps autoimmunity. 
Address correspondence to: Alan N. Houghton, Memorial Sloan-Kettering Cancer Center, 1275 York Avenue, New York, New York 10021, USA. Phone: (212) 639-7595; Fax: (212) 794-4352; E-mail: a-houghton@ski.mskcc.org.

1. Burnet, F.M. 1961. Immunological recognition of self. Science. 133:307-311.

2. Houghton, A.N., Gold, J.S., and Blachere, N.E. 2001. Immunity against cancer: lessons learned from melanoma. Curr. Opin. Immunol. 13:134-140.

3. Hahn, W.C., and Weinberg, R.A. 2002. Rules for making human tumor cells. N. Engl. J. Med. 347:1593-1603.

4. Land, H., Parada, L.F., and Weinberg, R.A. 1983. Tumorigenic conversion of primary embryo fibroblasts requires at least two cooperating oncogenes. Nature. 304:596-602.

5. Prehn, R.T., and Main, J.M. 1957. Immunity to methylcholanthrene-induced sarcomas. J. Natl. Cancer Inst. 18:769-778.

6. Lurquin, C., et al. 1989. Structure of the gene of tum- transplantation antigen P91A: the mutated exon encodes a peptide recognized with Ld by cytolytic T cells. Cell. 58:293-303.

7. Dubey, P., et al. 1997. The immunodominant antigen of an ultraviolet-induced regressor tumor is generated by a somatic point mutation in the DEAD box helicase p68. J. Exp. Med. 185:695-705.

8. Hewitt, H.B., Blake, E.R., and Walder, A.S. 1976. A critique of the evidence for active host defense against cancer, based on personal studies of 27 murine tumours of spontaneous origin. Br. J. Cancer. 33:241-259.

9. Naftzger, C., et al. 1996. Immune response to a differentiation antigen induced by altered antigen: a study of tumor rejection and autoimmunity. Proc. Natl. Acad. Sci. U. S. A. 93:14809-14814.

10. Weber, L.W., et al. 1998. Tumor immunity and autoimmunity induced by immunization with homologous DNA. J. Clin. Invest. 102:1258-1264.

11. Overwijk, W.W., et al. 1998. gp100/pmel 17 is a murine tumor rejection antigen: induction of "self"reactive, tumoricidal $\mathrm{T}$ cells using high-affinity, altered peptide ligand. J. Exp. Med. 188:277-286.

12. Bowne, W.B., et al. 1999. Coupling and uncoupling of tumor immunity and autoimmunity. J. Exp. Med. 190:1717-1722.

13. Gold, J.S., et al. 2003. A single heteroclitic epitope determines cancer immunity after xenogeneic DNA immunization against a tumor differentia- tion antigen. J. Immunol. 170:5188-5194.

14. Van Pel, A., Vessiere, F., and Boon, T. 1983. Protection against two spontaneous mouse leukemias conferred by immunogenic variants obtained by mutagenesis. J. Exp. Med. 157:1992-2001.

15. Boon, T., and Van Pel, A. 1978. Teratocarcinoma cell variants rejected by syngeneic mice: protection of mice immunized with these variants against other variants and against the original malignant cell line. Proc. Natl. Acad. Sci. U. S. A. 75:1519-1523.

16. Yu, Z., et al. 2004. Poor immunogenicity of a self/ tumor antigen derives from peptide-MHC-I instability and is independent of tolerance. J. Clin. Invest. 114:551-559. doi:10.1172/JCI200421695.

17. Houghton, A.N. 1994. Cancer antigens. Immune recognition of self and altered self. J. Exp. Med. 180:1-4.

18. Dyall, R., et al. 1998. Heteroclitic immunization induces tumor immunity. J. Exp. Med. 188:1553-1561.

19. Houghton, A.N., et al. 1985. Mouse monoclonal IgG3 antibody detecting GD3 ganglioside: a phase I trial in patients with malignant melanoma. Proc. Natl. Acad. Sci. U. S. A. 82:1242-1246.

20. Rasttter, W., Molina, A., White, C.A. 2004. Rituximab: expanding role in therapy for lymphomas and autoimmune diseases. Annu. Rev. Med. 55:477-503.

\title{
The T cell cometh: interplay between adaptive immunity and cytokine networks in rheumatoid arthritis
}

\author{
Gary S. Firestein
}

Division of Rheumatology, Allergy, and Immunology and Clinical Investigation Institute, University of California San Diego School of Medicine, San Diego, California, USA.

The etiology of autoimmunity in humans remains poorly defined, and animal models provide a unique opportunity to study potential autoimmune mechanisms. A novel model of autoimmune inflammatory arthritis results from a point mutation in the $\zeta$-associated-protein of $70 \mathrm{kDa}$ (ZAP-70), which causes abnormal thymic $T$ cell selection and survival of autoreactive clones (see the related article beginning on page 582). Although the resulting clinical and pathologic abnormalities are clearly $T$ cell-dependent, macrophage and fibroblast cytokines such as IL-1 and TNF- $\alpha$ are required for full expression of the disease. The studies of Hata et al. raise the intriguing possibility that traditional proinflammatory cytokine networks represent common effector mechanisms in inflammatory joint diseases such as rheumatoid arthritis. Hence, effective therapeutic interventions can target either unique etiologic pathways related to adaptive immune responses or shared terminal mechanisms.

Nonstandard abbreviations used: $\zeta$-associated protein of $70 \mathrm{kDa}$ (ZAP-70); collagen-induced arthritis (CIA); cyclic citrullinated peptide (CCP); Grb2-associated binder 2 (Gab2); linker for activation of T cells (LAT); lymphocyte protein tyrosine kinase (Lck); rheumatoid arthritis (RA); SH2-domain-containing leukocyte protein of $76 \mathrm{kDa}$ (SLP-76); Src homology 2 (SH2); vaccinia virus VH1-related (VHR).

Conflict of interest: The author has declared that no conflict of interest exists.

Citation for this article: J. Clin. Invest. 114:471-474 (2004). doi:10.1172/JCI200422651.
Like Sisyphus, who was doomed to forever push a boulder up a mountain only to see it roll down again, investigators attempting to develop models of rheumatoid arthritis (RA) have endured a frustrating existence. Many rodent strains engineered over the last few decades have been touted as "RA in a mouse (or rat)," and only later have the limitations and differences from human disease been recognized. Howev- er, with each model, we learn something new about the pathogenesis of synovial inflammation that can move the field one step closer to an effective therapy.

In this issue of the JCI, Hata and colleagues (1) have extended their insightful work on a novel inflammatory arthritis model resembling RA caused by a mutation in the gene encoding $\zeta$-associated protein of $70 \mathrm{kDa}$ (ZAP-70). In normal immune responses, CD4-associated lymphocyte protein tyrosine kinase (Lck) phosphorylates the $\xi$-chain of the $\mathrm{T}$ cell receptor-CD3 complex after antigen engagement (2). ZAP-70 is then recruited to the complex and is also phosphorylated by Lck (Figure 1). Dephosphorylation of inhibitory sites on ZAP-70 by low-molecular weight phosphotyrosine phosphatase further enhances ZAP-70 function. Several downstream targets subsequently serve as substrates for ZAP-70 kinase activity, including linker for activation of $\mathrm{T}$ cells (LAT), Grb2-associated binder 2 (Gab2), Src homology 2 (SH2) domain-containing leukocyte protein of 